\title{
Hybrid Multistarting GA-Tabu Search Method for the Placement of BtB Converters for Korean Metropolitan Ring Grid
}

\author{
Remund J. Labios, ${ }^{1}$ Seungjin Kim, ${ }^{2}$ Hwachang Song, ${ }^{2}$ and Byongjun Lee ${ }^{3}$ \\ ${ }^{1}$ Korea Electric Power Research Institute, Korea Electric Power Corporation, Daejeon 34056, Republic of Korea \\ ${ }^{2}$ Department of Electrical and Information Engineering, Seoul National University of Science er Technology, \\ Seoul 01811, Republic of Korea \\ ${ }^{3}$ School of Electrical Engineering, Korea University, Seoul 02841, Republic of Korea
}

Correspondence should be addressed to Hwachang Song; hcsong@seoultech.ac.kr

Received 3 December 2015; Accepted 7 April 2016

Academic Editor: Marek Lefik

Copyright (C) 2016 Remund J. Labios et al. This is an open access article distributed under the Creative Commons Attribution License, which permits unrestricted use, distribution, and reproduction in any medium, provided the original work is properly cited.

\begin{abstract}
This paper presents a method to determine the optimal locations for installing back-to-back (BtB) converters in a power grid as a countermeasure to reduce fault current levels. The installation of BtB converters can be regarded as network reconfiguration. For the purpose, a hybrid multistarting GA-tabu search method was used to determine the best locations from a preselected list of candidate locations. The constraints used in determining the best locations include circuit breaker fault current limits, proximity of proposed locations, and capability of the solution to reach power flow convergence. A simple power injection model after applying line-opening on selected branches was used as a means for power flows with BtB converters. Kron reduction was also applied as a method for network reduction for fast evaluation of fault currents with a given topology. Simulations of the search method were performed on the Korean power system, particularly the Seoul metropolitan area.
\end{abstract}

\section{Introduction}

For a highly complex power system such as that of the Korean metropolitan area, the increase in fault current levels due to an expanding network and an increasing number of load centers is a major concern for power system planners. The expansion of a power system leads to reduced Thévenin impedances and, thus, an increase in short-circuit fault current levels. One of the methods that can be taken to reduce fault current levels is the modification of the network topology or network reconfiguration, via bus-splitting and lineopening $[1,2]$. Line-opening, which will be the focus of this study, can be done by way of back-to-back (BtB) DC links.

The use of HVDC devices has been shown to aid in increasing and ensuring grid reliability and security $[3,4]$. This can be achieved by partitioning the network into sectors and interconnecting them with DC links instead of AC ties. The installation of HVDC devices, particularly back-to-back (BtB) converters, has also been shown to aid in the reduction of fault current levels [5]. When BtB converters are equipped on one end of a transmission line, the DC link decouples the line from the $\mathrm{AC}$ system, thus increasing the equivalent impedances of neighboring locations. The resultant increase in impedance can, therefore, aid in the reduction of fault current levels.

Partitioning AC systems into sectors by using DC interconnections also provides benefits simply by virtue of the nature of AC and DC systems: since DC systems do not provide reactive power, they effectively suppress the shortcircuit current of the connected $\mathrm{AC}$ system $[6,7]$. BtB converters, while in the category of HVDC systems, differ with DC transmission lines since the rectifiers and inverters are placed at the same substation. Also, the installation of BtB converters requires a relatively low cost compared to other HVDC systems.

However, installing a BtB DC link on a location or a combination of locations cannot be done easily due to several reasons: (a) a limited number of locations can only be selected due to the limitation of installation cost, (b) an operating point should still be maintained when any or all of the BtB 
links are activated (or opened), and (c) fault current limits imposed by existing equipment, such as circuit breakers, should not be exceeded. (Although existing circuit breakers can be rerated or replaced with new ones having a higherrating, this option may prove costly, especially when multiple locations are being considered.) Therefore, it is important to be able to determine the optimal locations where BtB converters are to be installed.

The use of fault level-constrained optimal power flow (FLC-OPF) has been studied in [8-11] as a method for conducting network reconfiguration for the reduction of fault current levels - a necessary step in operational planning. The network reconfiguration algorithm (NRA) proposed in these studies determines the locations where remedial actions, such as line-opening and bus-splitting, should be applied with the goal of reducing fault current levels. One limitation of this method, however, is that it consumes long computational runtimes. Additionally, performing contingency analysis on a power system proves difficult as it adds another layer of complexity for finding an optimal solution. To be able to overcome long computational times and accommodate contingency analysis so as to provide better solutions, faster search methods in the form of heuristic and metaheuristic techniques could be applied, and to be able to obtain good solutions that are specifically unique to our problem, a hybrid of some of these techniques can be formulated.

Real world problems, like those that exist in many aspects of power systems, require resolution of multiple objectives. Unlike strict mathematical methods, the use of hybrid metaheuristics has the ability to adapt to nonlinearities and discontinuities commonly found in power systems [12, 13]. References $[14,15]$ discuss the feasibility of applying various hybrid metaheuristic techniques to power systems, particularly in vulnerability assessment, system planning, power system scheduling, power system control, and solving OPF problems.

To improve upon the benefits of using metaheuristic techniques, hybridization of different techniques can be done by combining the complementary characteristics of different optimization strategies [16, 17]. Choosing an adequate combination of algorithmic concepts can, in fact, be the key for achieving top performance in solving many hard optimization problems. One example of hybridization is the use of local search methods within population-based methods. Population-based methods, like genetic algorithms (GA), are good in identifying promising areas of the search space in which local search methods, like tabu search, can then quickly determine the best solutions. Reference [18] outlines the characteristics of GA and tabu search and proposes ways on how the hybridization of these methods can be done. Studies of various hybridized tabu search methods for various applications [19-22] show the usefulness of the tabu search method, which is the basis of the algorithm proposed in this paper.

To solve the problem of reducing fault current levels by applying $\mathrm{BtB}$ converters while considering fault level constraints (FLCs), this study introduces a hybrid multistarting GA-tabu search method that solves the binary problem of whether or not a BtB converter is to be installed at a particular location (or locations) selected from a list of candidate locations. To find the best location or locations, the search method includes in its evaluation the following constraints: circuit breaker fault current limits, proximity of proposed locations, and capability of the solution to reach power flow convergence. The proposed algorithm is based mainly on tabu search for fast local search and is hybridized with GA and multistarting by adopting their concepts of elitism and improvement of diversity.

Power system planning requires the consideration of multiple combinations of cases and is further made difficult by the necessity of including fault current calculations for each case. Due to the combinatorial nature of this problem, a tabu search-based method was used in conjunction with some characteristics derived from multistarting and GA. (As mentioned in [18], GA does not excel in solving combinatorial problems.)

Discussed in Section 2 is the formulation of the problem, including the description of the power system used in the study, the BtB model applied, and the formulation of the objective function and its constraints. In Section 3, the issues in the development of the hybrid metaheuristic algorithm are described, including the network reduction method used to improve the speed of evaluation runtimes. The simulation results are shown in Section 4, and the conclusion of this paper is included in Section 5.

\section{Formulation of the Problem}

2.1. Formulation of the Objective Function. To determine whether or not a candidate location or a combination of multiple locations is suitable for the installation of a BtB converter, (1) is to be minimized:

$$
\begin{array}{ll}
\min & \sum_{i}^{N} w_{i} b_{i} \\
\text { s.t. } & \left|\frac{V_{k}}{Z_{k k}}\right| \leq I_{k, \max }, \quad k=1, \ldots, N,
\end{array}
$$

where $b_{i}$ the $i$ th decision variable, $w_{i}$ is weighting factor for $b_{i}, V_{k}$ is the voltage of bus $k, Z_{k k}$ is the $(k, k)$ element of impedance matrix, $I_{k, \max }$ is maximum allowed bus fault current, and $N$ is number of buses.

The decision variable, $b_{i}$, determines whether a candidate location is to be opened. The weighting factor, $w_{i}$, where a higher weight means a lower chance to be selected as a possible solution, is imposed onto the decision variable. The bus voltage, $V_{k}$, is calculated during fault occurrence while the decision variable is in effect. The impedance matrix of element $(k, k)$ is represented by the variable $Z_{k k}$. The maximum bus fault current is $I_{k, \max }$, and finally the total number of buses is represented by the variable $N$.

In addition to the original objective function (1), several constraints were also considered, and they are as follows:

(i) Fault level constraints, which are determined by circuit breaker ratings at specific bus locations, should be met.

(ii) Locations should not be adjacent (or share the same bus). 
(iii) Power flow analysis (while the decision variable is in effect) should converge.

These constraints were then added to the original objective function to form extended objective function (2), which is as follows:

$$
\begin{aligned}
\min & \sum_{i}^{N} w_{i} b_{i}+\sum_{i}^{N} P_{\mathrm{FLC}, i}+c_{\mathrm{ADJ}} b_{\mathrm{ADJ}}+c_{\mathrm{DIV}} b_{\mathrm{DIV}} \\
& +c_{\mathrm{CNT}} b_{\mathrm{CNT}},
\end{aligned}
$$

where

$$
\begin{aligned}
& P_{\mathrm{FLC}, i} \equiv \begin{cases}0, & I_{k} \leq I_{k, \text { max}}, \\
\left(I_{k}-I_{k, \text { max }}\right)^{2}, & I_{k}>I_{k, \text { max }},\end{cases} \\
& b_{\mathrm{ADJ}} \\
& \equiv \begin{cases}0, & \text { selected branches do not share a common bus, } \\
1, & \text { selected branches share a common bus, }\end{cases} \\
& b_{\mathrm{DIV}} \\
& \equiv \begin{cases}0, & \text { power flow converged (operating point is met), } \\
1, & \text { power flow diverged (operating point is not met), }\end{cases} \\
& b_{\mathrm{CNT}}, \\
& \equiv \begin{cases}0, & \text { power flow converged for any contingency case, } \\
1, & \text { power flow diverged for any contingency case; }\end{cases}
\end{aligned}
$$

$c_{\mathrm{FLC}}, c_{\mathrm{ADJ}}, c_{\mathrm{DIV}}$, and $c_{\mathrm{CNT}}$ are weighting factors.

2.2. Power System in Focus: The Seoul Metropolitan Area. This study focused on reducing fault current levels at the Seoul metropolitan area, which is a large and crucial portion of the Korean power system. Shown in Figure 1 is a representation of the Seoul metropolitan area, and it may be seen that the power network uses a system of loops interconnected by branches. These branches were used in the study as the candidate locations for the installation of BtB DC links. Among these candidate locations, the best locations (starting with a number of one until three) for BtB installation will be determined by the hybrid multistarting GA-tabu search method.

2.3. Power Injection Model for the BtB DC Link. To simulate the installation of a BtB DC link, the power injection model is used. First, a branch is to be opened. Afterwards, reactive powers of equal and opposite values are to be injected into the buses found at either end of the selected branch. Figure 2 shows a diagram of the model used in this study.

\section{The Hybrid Multistarting GA-Tabu Search Method}

3.1. Hybridization of Methods. Tabu search, on which the proposed hybrid algorithm is largely based, is a metaheuristic

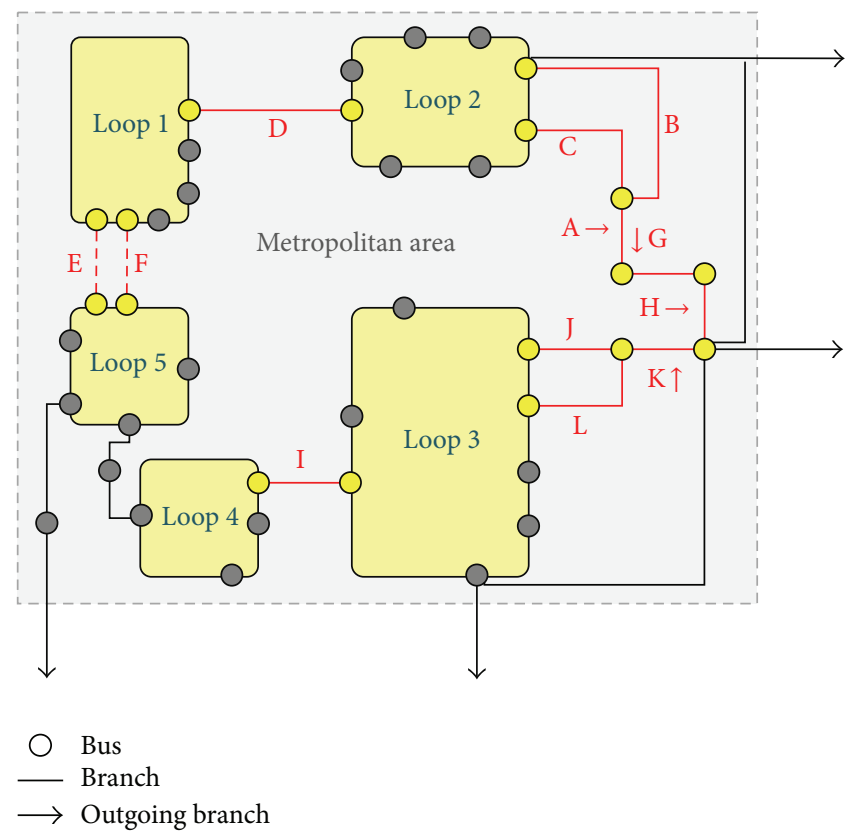

FIGURE 1: Representation of the Korean metropolitan ring grid used in the study. Letters A to $\mathrm{L}$ denote the location name.

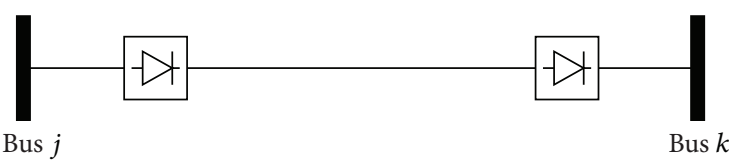

(a)
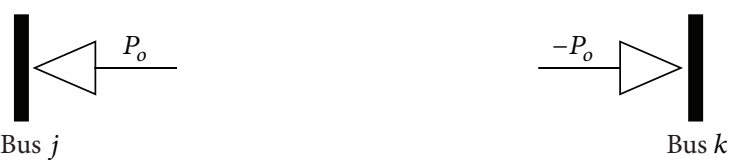

(b)

Figure 2: The BtB DC link in (a) can be represented as a simple power injection model as seen in (b).

technique commonly characterized as a local search method that uses a memory structure, called a tabu list. The tabu list deems solutions of lesser quality as forbidden and prevents them from being revisited for a duration of several iterations. Recency is a tabu search concept wherein a solution visited in the recent past is avoided, and tabu tenure is another concept that dictates the number of search iterations for a solution to remain forbidden. Another characteristic of tabu search that the proposed hybrid method uses is intensification, wherein the search is restarted with the best known solution.

Although GA is a popular metaheuristic technique used for its capability of exploring a wide solution space, it is trumped by tabu search when dealing with combinatorial problems. Due to its mutation and crossover features, GA has a tendency of losing solutions that may otherwise lead to finding much better solutions. However, the use of solution strings (or chromosomes) containing binary elements is a characteristic of GA that is useful in solving the problem 


\begin{tabular}{|l|l|l|l|l|l|l|l|l|l|}
\hline Solution string \\
\hline 1
\end{tabular}

(1) Candidate location with $\mathrm{BtB}$ converter

(0) Candidate location without $\mathrm{BtB}$ converter

FIGURE 3: Example of solution string to be used when considering 3 out of 10 candidate locations for BtB converter installation.

of whether or not a BtB converter is to be installed in a location. Another characteristic of GA that aligns with the intensification characteristic of tabu search is elitism, wherein the best solutions provide a starting point for finding the next best solution.

To improve diversity and allow the algorithm to evaluate more solutions, a multistarting approach was used. With restrictions in place to allow the algorithm to escape local minima, the multistarting approach in this study uses an adaptive long-term memory that prohibits restarting with a solution that has already been evaluated. This multistarting technique combined with the characteristics of GA and tabu search mentioned above are what constitute the proposed multistarting hybrid GA-tabu search method.

3.2. The Procedure of the Algorithm. The representation of a solution in the hybrid method is similar to that of GA where a population member (or solution) is comprised of a string of $1 \mathrm{~s}$ and 0 s. In the proposed hybrid method, a candidate location where a BtB converter is to be installed is represented by a " 1 ," while a candidate location that will retain its original setup is represented by a " 0 ." The total number of elements in a string is determined by the total number of candidate locations for $\mathrm{BtB}$ installation. Figure 3 shows an example of the scheme adopted in this study.

In order to have a controlled approach in the generation of the population of solutions, the total number of $1 \mathrm{~s}$ in a string should be equivalent to a predefined total number of locations where a $\mathrm{BtB}$ converter will be installed. This simple approach to the generation of the population is also a practical approach, as it will conduct a search based only on the desired number of $\mathrm{BtB}$ converter locations. (The user can perform separate searches for each desired total number of $\mathrm{BtB}$ converter locations.) The size of the initial population of solutions is calculated with the following equation:

$$
\text { Total no. of solutions }=\frac{n !}{r !(n-r) !},
$$

where $n$ is total number of candidate locations and $r$ is total number of locations for BtB converter installation.

From the initial population of solutions, a solution is selected randomly and is then evaluated for optimality with a tabu search-based method that performs a local search on neighboring solutions. The first neighboring solution is generated by a simple mutation of the previously selected solution string: a 1 bit is selected randomly and changed to a 0 bit, and then a 0 bit is selected randomly and changed to a 1 bit. The total number of 1 bits should be equal to $r$ in (4), as follows:

$$
\sum b_{i}=r
$$

This process is repeated until the desired tabu list size is met, and the best solution from this iteration is stored in a list of best solutions. All of the solutions that have been evaluated thus far are removed from the initial set of solutions, and those that remain will be used for the next restart.

The process is restarted multiple times, where the set of solutions is updated (i.e., reduced) during each restart until the maximum number of restarts is reached or until the set of solutions is exhausted. This multistarting technique ensures that a new starting solution is selected at each restart. Once the maximum number of restarts is reached, all solutions stored in the list of best solutions are compared. The solution with the least objective function will then be selected as the optimal solution. Figure 4 shows a flowchart of the algorithm.

After performing the tabu search once, the algorithm is to find a new starting point. For that purpose, the characteristics of the pure GA were adopted. It is noted that the solution for restarting the tabu search is just one and that the evaluation of objective function values takes quite a long time. The algorithm is to select two parents from the previous tabu search and then to randomly choose two bits for the crossover process. Next, the algorithm is to check whether two offspring solutions satisfy the equation of (5), and if there are one or two solutions not satisfying the condition, it performs the process to randomly compensate for excess or lacking bits. Of those solutions, the one with the better objective function value is provided to the next tabu search. There are two reasons why only two solutions are generated in the process. The first one is that it is to reduce the computational time, and the other is that the next tabu search can explore solutions that are farther away from the new starting point.

3.3. Network Reduction for Improved Evaluation Speed. To help reduce the evaluation time of the algorithm, Kron reduction (or node elimination) was done to reduce the network by eliminating nodes without current injection and retaining bus of special interest, as discussed in [23]. In this study, the buses of interest are those which are located within the Seoul metropolitan area.

Kron reduction is done through a "selective" application of Gaussian elimination by reducing the $Y_{\text {bus }}$ equations of the overall system to a set which contains only those bus voltages of special interest. All other buses are eliminated in the mathematical sense that their bus voltages and current injection do not appear explicitly.

\section{Numerical Results}

Shown in Table 1 is the system information of the 2027 Korean power system that was studied. Table 2 shows the fault level constraints of 33 circuit breaker locations used in the study. Simulations of the algorithm were performed in MATLAB $2011 \mathrm{~b}$ using a machine configured with the 64-bit version of 


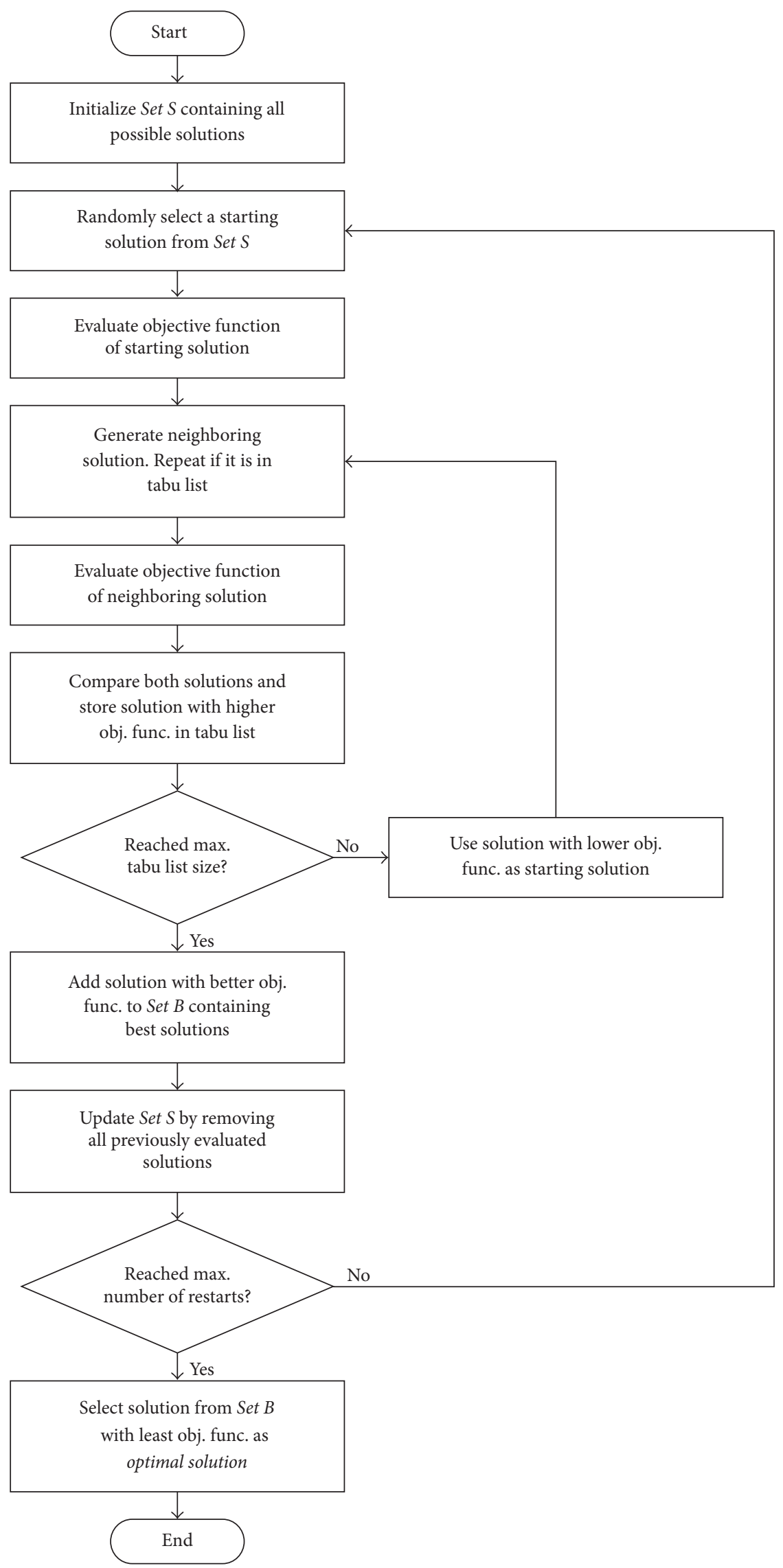

FIGURE 4: Flowchart of the proposed hybrid multistarting GA-tabu search method. 
TABLE 1: 2027 Korean power system information.

\begin{tabular}{lc}
\hline & Total number \\
\hline Buses & 2442 \\
Branches & 4105 \\
Generators & 368 \\
Buses with fault level constraints & 33 \\
Contingency cases & 11 \\
\hline
\end{tabular}

TABLE 2: Fault current limit of CBs.

\begin{tabular}{|c|c|}
\hline $\begin{array}{l}\text { Circuit breaker location } \\
\text { number }\end{array}$ & $\begin{array}{c}\text { Fault current limit } \\
(\mathrm{kA})\end{array}$ \\
\hline 1 & 50 \\
\hline 2 & 50 \\
\hline 3 & 50 \\
\hline 4 & 50 \\
\hline 5 & 50 \\
\hline 6 & 50 \\
\hline 7 & 50 \\
\hline 8 & 63 \\
\hline 9 & 50 \\
\hline 10 & 50 \\
\hline 11 & 63 \\
\hline 12 & 50 \\
\hline 13 & 50 \\
\hline 14 & 50 \\
\hline 15 & 50 \\
\hline 16 & 60 \\
\hline 17 & 60 \\
\hline 18 & 50 \\
\hline 19 & 50 \\
\hline 20 & 50 \\
\hline 21 & 50 \\
\hline 22 & 60 \\
\hline 23 & 50 \\
\hline 24 & 50 \\
\hline 25 & 50 \\
\hline 26 & 50 \\
\hline 27 & 63 \\
\hline 28 & 50 \\
\hline 29 & 50 \\
\hline 30 & 50 \\
\hline 31 & 63 \\
\hline 32 & 63 \\
\hline 33 & 50 \\
\hline
\end{tabular}

the Windows 7 operating system, an Intel ${ }^{\circledR}$ Core $^{\mathrm{TM}}$ i5-3470 $\mathrm{CPU}$ at $3.20 \mathrm{GHz}$, and $8.00 \mathrm{~GB}$ of RAM.

The tabu list size used in the simulation was the same as the total number of candidate locations. The tabu tenure counter was assigned a value of "1," giving the hybrid search
TABLE 3: Optimal locations for BtB DC link installation.

\begin{tabular}{lcccccc}
\hline Location/branch & \multicolumn{6}{c}{ Number of BtB DC links to be installed } \\
name & 1 & 2 & 3 & 4 & 5 & 6 \\
\hline A & & & & & & O \\
B & & & & & & \\
C & & O & O & O & O \\
D & O & O & & O & \\
E & & & & & & O \\
F & & & & O & O & O \\
G & & & & & O & \\
H & & & & O & & O \\
I & & O & & & \\
J & & & O & O & O \\
K & & & & & & \\
L & & & & & & \\
\hline
\end{tabular}

TABLE 4: Simulation elapsed time before and after applying Kron reduction.

\begin{tabular}{|c|c|c|c|}
\hline \multirow[b]{2}{*}{$\begin{array}{l}\text { Number of } \\
\text { locations }\end{array}$} & \multicolumn{2}{|c|}{ Total simulation time } & \multirow[b]{2}{*}{ Percent reduction } \\
\hline & $\begin{array}{l}\text { Without Kron } \\
\text { reduction } \\
(\mathrm{sec})\end{array}$ & $\begin{array}{l}\text { With Kron } \\
\text { reduction } \\
(\mathrm{sec})\end{array}$ & \\
\hline 1 & 3383.08 & 700.00 & $79.31 \%$ \\
\hline 2 & 17592.38 & 3247.16 & $81.54 \%$ \\
\hline 3 & 19183.40 & 7859.34 & $59.03 \%$ \\
\hline 4 & 14877.60 & 1302.90 & $91.24 \%$ \\
\hline 5 & 12890.59 & 1266.48 & $90.18 \%$ \\
\hline 6 & 12256.85 & 469.06 & $96.17 \%$ \\
\hline
\end{tabular}

algorithm a short-term memory that allows it to revisit and reevaluate previous solutions.

Prior to performing the hybrid search method, the following parameters would first have to be set:

(i) Candidate locations (or branches) to be evaluated for line-opening.

(ii) The total number of candidate locations to be opened.

(iii) Values of weighting factors to be used for the objective function.

(iv) Number of restarts to be done by the hybrid search method.

(v) Number of neighboring solutions to be generated.

(vi) Method to be used for power flow analysis (NewtonRaphson, fast-decoupled) and tolerances to be applied.

(vii) Option on whether or not to include N-1 contingencies in the evaluation process.

(viii) Option on whether or not to use the DC injection model in the evaluation process.

Table 3 shows the optimal locations for installing the BtB converters. Figure 4 shows the variation in optimal objective 


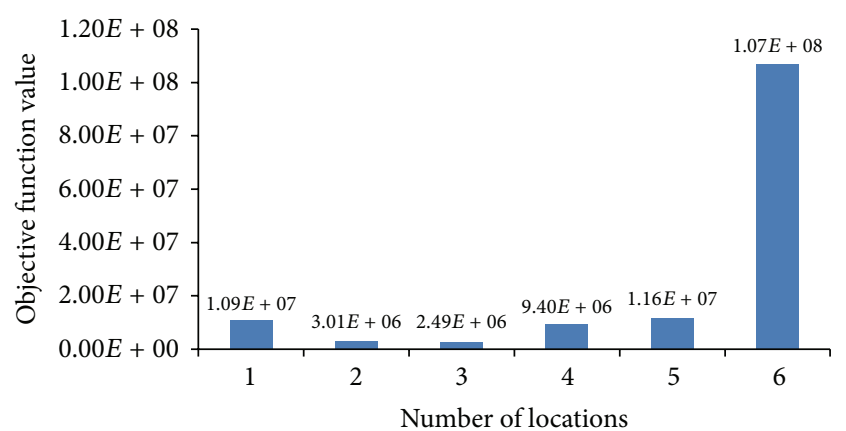

FIGURE 5: Objective function values as desired number of $\mathrm{BtB}$ locations is increased.

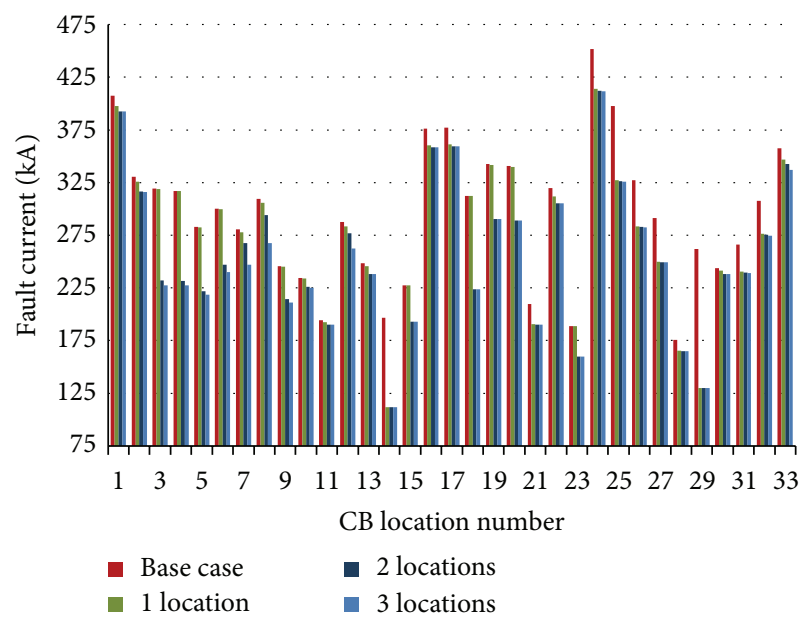

Figure 6: Fault current (kA) at each circuit breaker location (1 to 3 locations).

function values as the number of locations for installing $\mathrm{BtB}$ converters is changed. Figures 5 and 6 show the reduction of fault current levels while the BtB converters are in effect during fault occurrence.

It may be seen in Figure 5 that there is a marked difference in the objective function values when more than one location is installed with a BtB converter. However it may also be observed that an increase in the number of locations for installing $\mathrm{BtB}$ converters does not always result in an improvement in the objective function value. As an example, referring to Figure 5, although having 4 or 5 lineopening locations may reduce fault current levels, the amount by which they are reduced are not as good as having only 2 or 3 line-opening locations, or on the other hand, as in the case of 6 line-opening locations, a solution that also meets most, if not all, of the contingency cases cannot be found.

Figure 6 shows that fault current levels were successfully reduced. It may be seen that installation of BtB converters at the optimal locations can reduce fault current levels.

Shown in Table 4 is the simulation elapsed time prior to applying Kron reduction, as well as the improvements in simulation elapsed time after Kron reduction was applied. It may be seen from the results that there is a significant

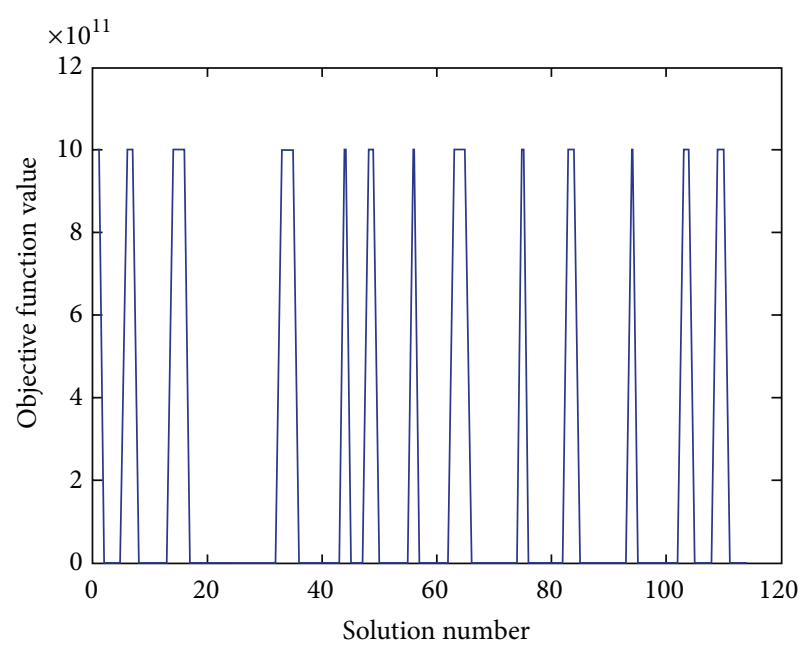

FIGURE 7: Objective function values of evaluated solutions when searching for the best 3 locations for BtB installation.

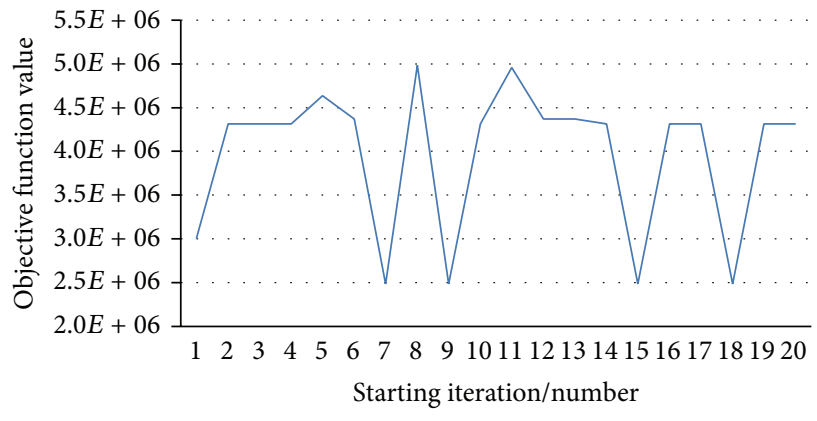

FIGURE 8: Objective function values of the best found solutions in each iteration for one full run when searching for the best 3 locations for BtB installation.

decrease in the elapsed time. The time spent for the search procedure in the hybrid algorithm is negligible, compared to that for the evaluation of utility function values.

To provide an example of the evaluation process, Figure 7 shows the change in the objective function throughout the whole search process. An objective function that reaches a value of about $1 \times 10^{12}$ means that the solution being evaluated has been assigned with a high weight, which effectively removes that solution from being selected as an optimal solution. Figure 7 also shows the stochastic nature of the search method, wherein each run of the search may result in a different optimal solution. This reaffirms the necessity for applying a multistarting technique to allow more solutions to be evaluated and increase the probability of finding the best solution.

Figure 8 shows the best found solutions after the maximum number of starts has been met (a total of 20, in this case). The varying results in each iteration shows that multistarting is needed to be able to find the optimal solution for the problem. It can be seen in the figure that the optimal solution for the problem was found in the 7 th iteration or start and was found three additional times hence. 
The main concern was to find an acceptable solution within a reasonable time frame. For this purpose, a hybrid search method was proposed in the paper with the intention of reducing the number of objective function evaluations performed. The controlling parameters in tabu search are tabu list size and tabu tenure counter. The change in tabu tenure counter is quite sensitive in terms of the time spent in completing the search method. That change possibly increases the number of objective function evaluations because it might lead to the lack of diversity. In the case of tabu list size, setting it to the number of candidate locations is recommended.

\section{Conclusion}

In this paper, a hybrid multistarting GA-tabu search method was used to successfully determine the best locations to install $\mathrm{BtB}$ converters to help reduce fault current levels in a power system. It has also been shown that the installation of BtB converters in a power system is a viable option when considering what countermeasures are to be applied in a power system during the occurrence of a fault. Furthermore, it may be concluded that applying Kron reduction to the network prior to conducting the search can significantly decrease the simulation time.

The authors recommend that further studies be performed to further improve the search method used in this study. One aspect that can be improved on is the ability of the algorithm to handle large networks at a reasonable computational cost while satisfying a considerable number of constraints. Other search methods are also suggested to be used in conjunction with the hybrid GA-tabu search method of this study.

\section{Competing Interests}

The authors declare that there are no competing interests regarding the publication of this paper.

\section{Acknowledgments}

This research was supported by Basic Science Research Program through the National Research Foundation of Korea (NRF) funded by the Ministry of Education (no. 2014R1A1A2056806) and by the Human Resources Development of the Korea Institute of Energy Technology Evaluation and Planning (KETEP) grant funded by the Korea government Ministry of Trade, Industry \& Energy (no. 20154030200720).

\section{References}

[1] N. Tleis, Power Systems Modelling and Fault Analysis: Theory and Practice, Elsevier, New York, NY, USA, 2008.

[2] G. Andersson, Modelling and Analysis of Electric Power Systems, ETH Zürich, Zürich, Switzerland, 2008.

[3] V. K. Sood, HVDC and FACTS Controllers: Applications of Static Converters in Power Systems, Kluwer, New York, NY, USA, 2004.
[4] C. K. Kim, V. K. Sood, G. Jang, S. J. Lim, and S. J. Lee, HVDC Transmission: Power Conversion Applications in Power Systems, John Wiley \& Sons Asia, Singapore, 2009.

[5] H. Song, S. Ohn, and B. Jang, "Enhancement of interface flow margins using back-to-back converters in power systems," International Review of Electrical Engineering, vol. 7, no. 5, pp. 5678-5685, 2012.

[6] H. K. Clark, A.-A. Edris, M. M. El-Gasseir, H. D. K. Epp, A. Isaacs, and D. Woodford, "Softening the blow of disturbances," IEEE Power and Energy Magazine, vol. 6, no. 1, pp. 30-41, 2008.

[7] M. Yoon, J. Park, and G. Jang, "A study of HVDC installation in Korean capital region power system," in Proceedings of the IEEE Power and Energy Society General Meeting (PES '12), San Diego, Calif, USA, July 2012.

[8] P. N. Vovos, G. P. Harrison, A. R. Wallace, and J. W. Bialek, "Optimal power flow as a tool for fault level-constrained network capacity analysis," IEEE Transactions on Power Systems, vol. 20, no. 2, pp. 734-741, 2005.

[9] P. N. Vovos and J. W. Bialek, "Direct incorporation of fault level constraints in optimal power flow as a tool for network capacity analysis," IEEE Transactions on Power Systems, vol. 20, no. 4, pp. 2125-2134, 2005.

[10] P. Vovos, H. Song, K.-W. Cho, and T.-S. Kim, "A network reconfiguration algorithm for the reduction of expected fault currents," in Proceedings of the IEEE Power \& Energy Society General Meeting (PES '13), Vancouver, Canada, July 2013.

[11] H. Song, P. Vovos, T.-S. Kim, and K.-W. Cho, "Decision making on bus splitting locations using a modified fault current constrained optimal power flow (FCC-OPF)," Journal of Electrical Engineering \& Technology, vol. 11, no. 1, pp. 76-85, 2016.

[12] S. Voss, "Metaheuristics," in Encyclopedia of Optimization, C. A. Floudas and P. M. Pardalos, Eds., vol. 3, pp. 2061-2074, Springer, New York, NY, USA, 2nd edition, 2009.

[13] F. Glover and M. Laguna, Tabu Search, Kluwer Academic Publishers, Boston, Mass, USA, 1997.

[14] K. Y. Lee and M. A. El-Sharkawi, Eds., Modern Heuristic Optimization Techniques: Theory and Applications to Power Systems, John Wiley \& Sons, Hoboken, NJ, USA, 2008.

[15] S. A. Soliman and A. H. Mantawy, Eds., Modern Optimization Techniques with Applications in Electric Power Systems, Springer, New York, NY, USA, 2012.

[16] C. Blum, J. Puchinger, G. R. Raidl, and A. Roli, "Hybrid metaheuristics in combinatorial optimization: a survey," Applied Soft Computing, vol. 11, no. 6, pp. 4135-4151, 2011.

[17] I. Boussaïd, J. Lepagnot, and P. Siarry, "A survey on optimization metaheuristics," Information Sciences, vol. 237, pp. 82-117, 2013.

[18] F. Glover, J. P. Kelly, and M. Laguna, "Genetic algorithms and tabu search: hybrids for optimization," Computers and Operations Research, vol. 22, no. 1, pp. 111-134, 1995.

[19] Y. Wang, J.-K. Hao, F. Glover, and Z. Lü, "A tabu search based memetic algorithm for the maximum diversity problem," Engineering Applications of Artificial Intelligence, vol. 27, pp. 103$114,2014$.

[20] F. Glover, T. Ye, A. P. Punnen, and G. Kochenberger, "Integrating tabu search and VLSN search to develop enhanced algorithms: a case study using bipartite boolean quadratic programs," European Journal of Operational Research, vol. 241, no. 3, pp. 697-707, 2015.

[21] L. Lasdon, A. Duarte, F. Glover, M. Laguna, and R. Martí, "Adaptive memory programming for constrained global optimization," Computers \& Operations Research, vol. 37, no. 8, pp. 1500-1509, 2010. 
[22] Y. A. Katsigiannis, P. S. Georgilakis, and E. S. Karapidakis, "Hybrid simulated annealing-tabu search method for optimal sizing of autonomous power systems with renewables," IEEE Transactions on Sustainable Energy, vol. 3, no. 3, pp. 330-338, 2012.

[23] A. R. Bergen and V. Vittal, Power Systems Analysis, Prentice Hall, Upper Saddle River, NJ, USA, 2nd edition, 2000. 


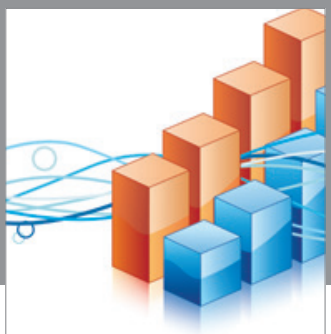

Advances in

Operations Research

vatem alat4

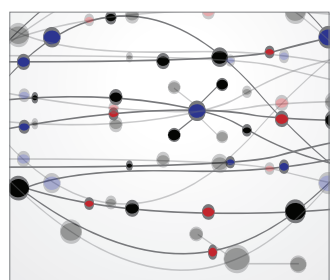

\section{The Scientific} World Journal
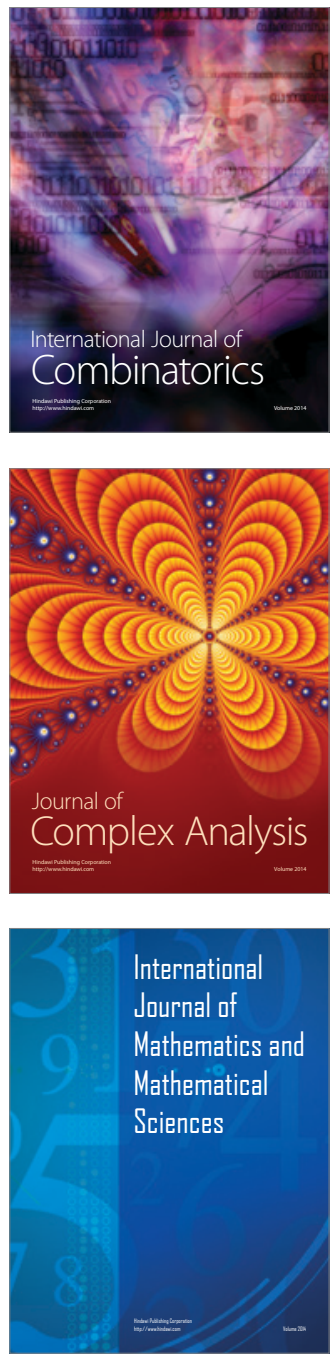
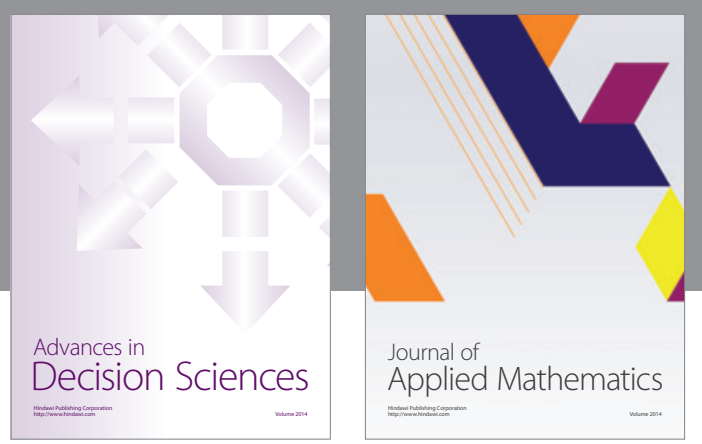

Algebra

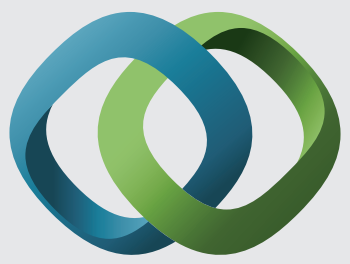

\section{Hindawi}

Submit your manuscripts at

http://www.hindawi.com
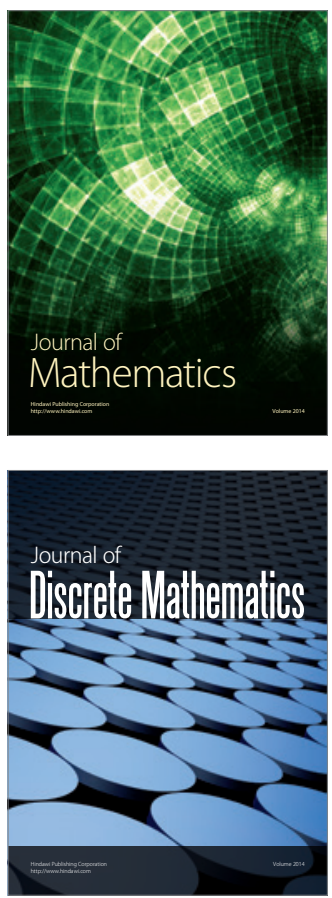

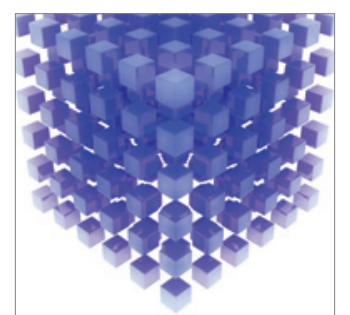

Mathematical Problems in Engineering
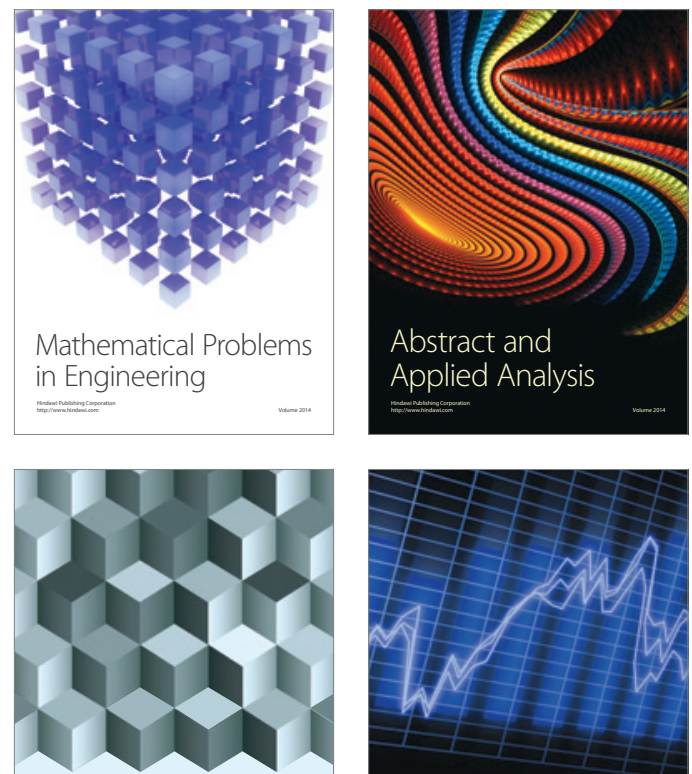

Journal of

Function Spaces

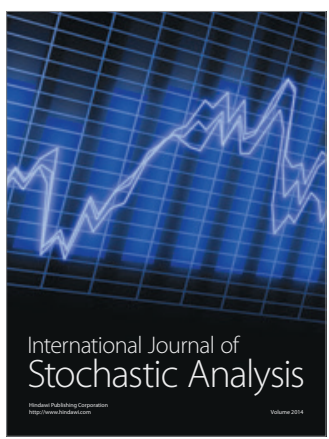

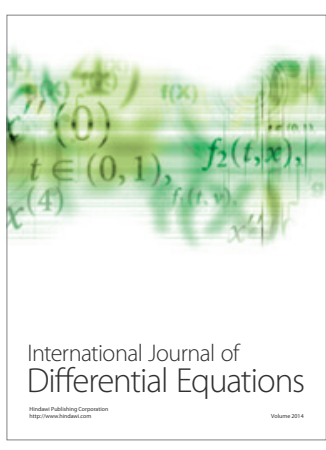
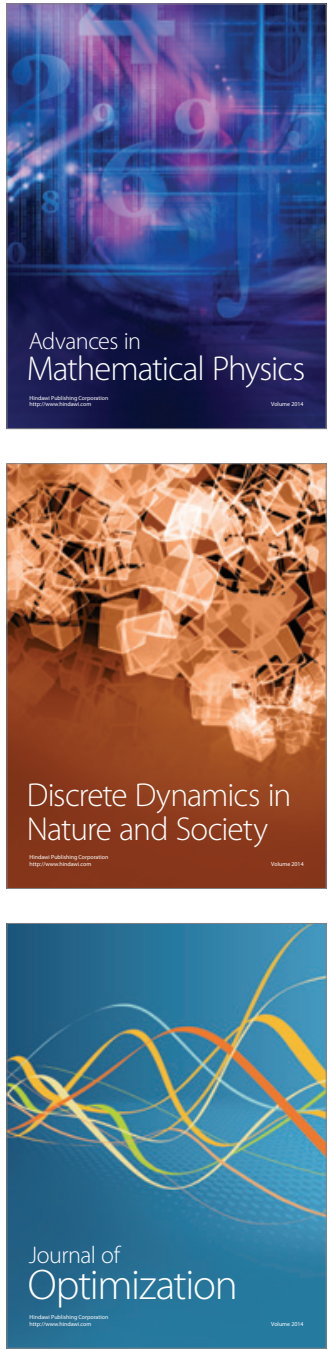